\section{Conservative treatment of retinoblastoma: a prospective phase II randomized trial of neoadjuvant chemotherapy followed by local treatments and chemothermotherapy}

${ }^{1}$ Department of Ocular Oncology, Institut Curie, Paris, France

${ }^{2}$ Department of Pediatric, Adolescent and Young Adults Oncology, Institut Curie, Paris, France

${ }^{3}$ Department of Biostatistics, Institut Curie, Paris, France

${ }^{4}$ Department of Pediatric Oncology, CHU Toulouse, Hôpital des Enfants, Toulouse, France

${ }^{5}$ Department of Anaesthesiology, Institut Cure, Paris, France

${ }^{6}$ University Paris Descartes, Sorbonne Paris Cité, Paris, France

Correspondence:

L Lumbroso-Le Rouic, Department of Ocular Oncology, Institut Curie, 26 rue d'Ulm, 75005 Paris, France

Tel: +33 1 44324602;

Fax: +33153104018

E-mail: livia.lumbroso@ curie.fr

Received: 14 April 2015 Accepted in revised form: 3 August 2015

Published online:

2 October 2015

\begin{abstract}
Purpose Intraocular retinoblastoma treatments often combine chemotherapy and focal treatments. A first prospective protocol of conservative treatments in our institution showed the efficacy of the use of two courses of chemoreduction with etoposide and carboplatin, followed by chemothermotherapy using carboplatin as a single agent and diode laser. In order to decrease the possible longterm toxicity of chemotherapy due to etoposide, a randomized neoadjuvant phase II protocol was conducted using vincristinecarboplatin $v s$ etoposide-carboplatin.

Patients and methods The study was proposed when initial tumor characteristics did not allow front-line local treatments. Patients included in this phase II noncomparative randomized study of neoadjuvant chemotherapy received vincristin-carboplatin (new arm) vs etoposide-carboplatin (our reference arm). They were subsequently treated by local treatments and chemothermotherapy. Primary end point was the need for secondary enucleation or external beam radiotherapy (EBRT) not exceeding $40 \%$ at 2 years. Results A total of 65 eyes in 55 children were included in the study (May 2004 to August 2009). Of these, 32 eyes (27 children) were treated in the arm etoposide-carboplatin and 33 eyes ( 28 children) in the arm vincristin-carboplatin. At 2 years after treatment, 23/33 (69.7\%) eyes were treated and salvaged without EBRT or enucleation in the
\end{abstract}

arm vincristin-carboplatin and $26 / 32(81.2 \%)$ in the arm etoposide-carboplatin.

Conclusion Even if the two treatment arms could be considered as sufficiently active according to the study decision rules, neoadjuvant chemotherapy by two cycles of vincristine-carboplatin followed by chemothermotherapy appear to offer less optimal local control than the etoposidecarboplatin combination.

Eye (2016) 30, 46-52; doi:10.1038/eye.2015.179; published online 2 October 2015

\section{Introduction}

The conservative management of retinoblastoma requires a combination of several treatment modalities in order to obtain tumor control, preservation of the eye, and to optimize visual function. Radiotherapy (external beam or radioactive plaque brachytherapy) was the first main conservative treatment. Then treatment regimens comprising chemotherapy appeared in order to avoid the well-known adverse effects of external beam radiotherapy (EBRT) (especially the increased risk of secondary tumor in the irradiation field). ${ }^{1,2}$ Numerous conservative treatment modalities have been published: cryoapplication, thermotherapy alone or combined with systemic chemotherapy, plaque radiotherapy, or even exclusive chemotherapy. ${ }^{3-12}$ More recently, periocular, intraarterial, and intravitreal administration of chemotherapy have been used and are still 
under evaluation. These last modalities were developed during the study period. ${ }^{13-15}$ The current conservative strategy often needs the combination of several of the therapeutic modalities indicated above. Our team published, in 2008, a phase II prospective study on the efficacy of a treatment combining local therapies or chemothermotherapy after chemoreduction by etoposide and carboplatin ( 2 cycles) whenever the tumors are not directly treatable by local treatments. ${ }^{8}$ This study confirmed the validity of our proposed approach. Tumor control rates were high and comparable to those reported in other studies, and to the results of the reference treatment, that is, EBRT. The cumulative doses of chemotherapy used for the majority of patients were lower than those reported in most other publications and the immediate safety was excellent. Chemotherapy is obviously associated with a possible long-term mutagenic risk, especially after using etoposide in children often presenting a constitutional mutation of the $R b 1$ gene, even if etoposide-induced secondary leukemia has been only very rarely reported after retinoblastoma treatment and always after higher cumulative doses of $>2 \mathrm{~g} / \mathrm{m}^{2} .{ }^{16}$ The next step was to discuss a less intensive therapy in order to decrease the possible toxicities, and a phase II randomized study has been realized where patients who required neoadjuvant treatment received vincristine+carboplatin (new arm of this study) vs carboplatin+etoposide (our reported standard arm). This treatment was followed by the local therapies usually used in our center. The results of this study are presented in this paper.

\section{Patients and methods}

This was a multicenter study, designed as a phase II, prospective, randomized, noncomparative evaluation of two cycles of neoadjuvant chemotherapy before the different modalities used for the conservative treatment of retinoblastoma, such as chemothermotherapy, thermotherapy, cryoapplication, and plaque brachytherapy (all the local ocular treatments and evaluations were conducted in Institut Curie).

The primary objective was to evaluate the tumor response after vincristine-carboplatin and after etoposide-carboplatin. The main objective was to obtain a percentage of conservatively treated eyes without EBRT of $\geq 60 \%$, as obtained by our standard approach used (etoposide-carboplatin). ${ }^{8}$ Secondary objectives were evaluation of the short, medium-, and long-term systemic adverse effects, particularly the development of second tumors.

All patients with unilateral or bilateral retinoblastoma (without age limit) amenable to conservative management in at least one eye for bilateral cases and requiring an initial neoadjuvant chemotherapy were eligible for this study. Patients bearing tumors not occupying more of half of the vitreous cavity and without invasion of the anterior segment of the eye or extraocular involvement. For bilateral retinoblastoma patients with one eye requiring first-line enucleation and conservative treatment in the other eye, the enucleated eye was not included in the analysis. Patients harboring only tumors that were directly amenable to local ophthalmic treatments or chemothermotherapy (ie, tumors of $<3 \mathrm{~mm}$ ) were not included.

According to current guidelines and legislation in France, this interventional study was approved by an independent ethics committee. The study sponsor had obtained an appropriate civil liability insurance policy and both parents signed an informed consent before inclusion in the protocol.

\section{Treatments}

For all children, an ocular fundus examination under general anesthesia was performed by one of the three senior ophthalmologists. An ocular fundus diagram was established and the Reese-Ellsworth classification as well as the International Classification of Retinoblastoma $(\mathrm{ICRB})^{17}$ were determined.

Clinical pediatric examination was performed as well as imaging (MRI more often than CT scan) of the orbits and brain.

Following this examination, inclusion in the study was proposed when it was envisaged to preserve at least one eye and when neoadjuvant chemotherapy was indicated before the ocular treatments.

After consent of parents, randomization was realized by the clinical investigations unit, and the child received either vincristine-carboplatin or our standard etoposidecarboplatin combination.

\section{Neoadjuvant chemotherapy phase}

Reference arm Children over the age of 1 year or weighing $>10 \mathrm{~kg}$ received etoposide $150 \mathrm{mg} / \mathrm{m}^{2} /$ day from day 1 to day 3 (D1 to D3) and carboplatin $200 \mathrm{mg} / \mathrm{m}^{2} /$ day from D1 to D3 using a 1-h infusion for each drug. In children under 1 year and/or weighing $<10 \mathrm{~kg}$, chemotherapy doses were etoposide $5 \mathrm{mg} / \mathrm{kg}$ / day and carboplatin $6.7 \mathrm{mg} / \mathrm{kg} /$ day from D1 to D3. A further dose reduction was used in children between 1 and 2 months: etoposide $3.3 \mathrm{mg} / \mathrm{kg} /$ day and carboplatin $4.5 \mathrm{mg} / \mathrm{kg} /$ day from D1 to D3.

New arm of the study In children over the age of 1 year and weighing $>10 \mathrm{~kg}$, carboplatin $560 \mathrm{mg} / \mathrm{m}^{2} /$ day (in 1-h infusion) and vincristine $1.5 \mathrm{mg} / \mathrm{m}^{2} /$ day (bolus infusion), maximal dose $2 \mathrm{mg}$ was administered at D1. 
In children under 1 year and/or $<10 \mathrm{~kg}$, carboplatin doses were adjusted to the weight, $18.7 \mathrm{mg} / \mathrm{kg} /$ day at D1 and vincristine $0.05 \mathrm{mg} / \mathrm{kg} /$ day at D1.

In children from 1 to 2 months the vincristine dose was unchanged $(0.05 \mathrm{mg} / \mathrm{kg} /$ day $)$ but a further carboplatin dose reduction was used $(12.5 \mathrm{mg} / \mathrm{kg} /$ day $)$.

In neonates until the age of 1 month we used the same interim chemotherapy than previously reported with two courses of cyclophosphamide $10 \mathrm{mg} / \mathrm{kg}$ /day from D1 to D3 and vincristine $0.05 \mathrm{mg} / \mathrm{kg}$ on D1 at 2 weeks of interval before starting vincristine-carboplatin or etoposide-carboplatin neoadjuvant chemotherapy. ${ }^{8}$

After the second course of chemotherapy, ocular fundus examination under general anesthesia was performed for clinical complete ocular tumor evaluation and to guide the following therapies for each tumor. Conservative treatment was considered a success when only chemothermotherapy, thermotherapy, cryoapplication, or iodine $125\left({ }^{125} \mathrm{I}\right)$ plaque brachytherapy were used. The need for EBRT or enucleation when the tumor was still not amenable to conservative management were considered as failures.

\section{Ophthalmic therapy}

The various treatment modalities proposed after the initial chemoreduction phase (two cycles) are chemothermotherapy (CTT), cryoapplication, thermotherapy, and ${ }^{125} \mathrm{I}$ brachytherapy, with the indications adopted by our center and described elsewhere. $^{8}$

For chemothermotherapy, the technique consists a combination of transpupillary thermotherapy (TTT) delivered after systemic administration of carboplatin. A dose of $560 \mathrm{mg} / \mathrm{m}^{2}$ is administered intravenously 1 to $2 \mathrm{~h}$ before thermotherapy. For children weighing $<10 \mathrm{~kg}$ or younger than 12 months, the dose is adapted to weight $(18.7 \mathrm{mg} / \mathrm{kg})$. TTT is then delivered under general anesthesia with fully dilated pupils. A continuous mode diode laser $(810 \mathrm{~nm})$ (Iridex, Mountain View, CA, USA) is used with a microscope adapter allowing a spot size ranging from 0.3 to $2.0 \mathrm{~mm}$. Laser hyperthermia (thermotherapy) is applied under the operating microscope and through a contact lens (three-mirror lens). Each tumor is treated separately in a single session. This cycle is administered every 28 days for a minimum of 1 to a maximum of 6 cycles.

These various modalities may be combined for the treatment of one or several tumors for each eye and child.

\section{Follow-up}

Ophthalmologic After treatment, monthly assessment by ocular fundus examination under general anesthesia is performed for the first year, and the frequency of followup is then gradually reduced to a quarterly examination. Ocular fundus photographs are regularly performed with the Retcam (Clarity Medical Systems, Inc., Pleasanton, CA, USA).

Long-term follow-up is performed every 3 months for several years on an outpatient basis.

Pediatric During treatment: Children were closely followed for the management of chemotherapy toxicity (digestive, hematologic, infectious), including laboratory assessments (complete blood count, 2 to 3 times a week and serum electrolytes, urea, creatinine, liver function tests, before each course). Hearing assessment was performed initially and during follow-up. ${ }^{18}$

After treatment: Clinical follow-up is realized every 2 months after completion of treatments, then every 6 months for 1 year and then annually. It includes interview and complete clinical examination, evaluation of growth, as well as auditory and renal function and screening for second tumors.

\section{Statistical methods}

The primary end point of this study was the need for EBRT or enucleation (defined as failures of the treatment) at 2-year follow-up. Missing outcomes were considered as failures.

Because the failure rate observed with the reference treatment (etoposide-carboplatin) was not known with sufficient accuracy, a randomized phase II trial was planned, with etoposide-carboplatin arm being an internal reference to validate the hypothesis used to estimate the sample size. Because a usual randomized study, designed to detect a small difference in outcomes between the two arms, would have needed a large number of patients (not compatible with a phase II study nor a rare disease), no formal statistical comparison between the two arms was planned. . $^{190}$

The decision rules were the same for our reference (etoposide-carboplatin) and the new arm (vincristinecarboplatin), and were defined according to the singlestage Fleming design. ${ }^{21,22}$ The number of eyes to include in each arm is 33 (17 to 33 patients). If $\geq 11$ failures are observed among the 33 eyes $(\geq 33.3 \%)$, the percentage of failures is considered as superior to what was expected, and corresponding treatment is considered uninteresting for further evaluation. If failures are $\leq 10(\leq 30.3 \%)$, the percentage of failures is considered sufficiently low to conclude that corresponding treatment is active. Based on previous experience, ${ }^{8}$ the null hypothesis was defined as a failure rate of $\geq 40 \%$ vs an alternative hypothesis of a failure rate of $\leq 20 \%$. With the above decision rules, the probability to conclude that a treatment is effective if the 
failures are $\leq 20 \%$ (ie, power in each arm) is $95 \%$. The probability to conclude that it is effective if the failures are $\geq 40 \%$ (ie, type I error in each arm) is $16.9 \%$.

In order to stop the study earlier if the failures rate is greater than expected, a futility interim analysis was planned. Among the first 13 eyes included in each arm (7 to 13 patients), if $\geq 4$ failures were observed at 1 -year follow-up, accrual to the corresponding arm would be terminated. This rule allows stopping accruals in $>85 \%$ of cases if failures rate is $>50 \%$.

Because of the nature of disease under evaluation, subjects could provide information for only one or both eyes. Thus, two outcomes could be observed in the same individual. Because of the small sample size of the study, no particular technique was implemented to take into account correlation between the outcomes observed within one subject.

\section{Results}

During the study period (May 2004 to August 2009), 55 children (65 eyes) were recruited in the study. In the same time period, 304 children were referred for retinoblastoma in our center. Of these, 249 were not included ( $242 \mathrm{did}$ not have inclusion criteria, 1 parent refusal, and 6 children for whom the follow-up was doubtful; see Supplementary Figure 1 flow chart online).

Median follow-up at the time of analysis is 4.2 years in each arm. A total of 27 children (32 eyes) were randomized as per standard treatment: etoposidecarboplatin. Mean age at inclusion was 11 months, and male/female sex ratio was 13:14. A total of 28 children (33 eyes) were randomized as 'new treatment': vincristine-carboplatin.

None of the children were seen under the age of 1 month, and none of them received cyclophosphamide. Mean age at inclusion was 11.9 months, and male/female sex ratio was 15:13. Both arms were also comparable in terms of unilaterality or bilaterality of cases.

All subsequent analyses were realized in terms of eyes included in each arm (ie, 32 eyes randomized in the standard arm and 33 eyes randomized in the experimental arm). Initial characteristics of eye classification according to the Reese-Ellsworth and IRC classifications and presence of subretinal or vitreous seeding are described in Tables 1,2,3. Group D and secondary subretinal seeding were more frequent in the experimental arm.

After the initial randomized phase of two cycles of chemoreduction, the children are treated according to our conservative standard treatments. Each type of local treatment is described in Table 4. They are comparable in each arm.
Table 1 Description of Reese-Ellsworth classification: number of eyes $(\%)$

\begin{tabular}{lcc}
\hline Reese & Etoposide-carboplatin & Vincristine-carboplatin \\
\hline I & $7(21.9 \%)$ & $4(12.1 \%)$ \\
II & $9(28.1 \%)$ & $6(18.2 \%)$ \\
III & $10(31.5 \%)$ & $15(45.5 \%)$ \\
IV & $2(6.2 \%)$ & $3(9.1 \%)$ \\
V & $4(12.5 \%)$ & $5(15.2 \%)$ \\
\hline
\end{tabular}

Table 2 IRC classification: number of eyes (\%)

\begin{tabular}{lcc}
\hline IRC classification & Etoposide-carboplatin & Vincristine-carboplatin \\
\hline A & $2(6.2 \%)$ & $1(3.0 \%)$ \\
B & $16(50 \%)$ & $16(48.5 \%)$ \\
C & $7(21.9 \%)$ & $4(12.1 \%)$ \\
D & $7(21.9 \%)$ & $12(36.4 \%)$ \\
\hline
\end{tabular}

Table 3 Presence of subretinal or vitreous seeding eyes (\%)

\begin{tabular}{lcc}
\hline Seeding & $\begin{array}{c}\text { Etoposide- } \\
\text { carboplatin }\end{array}$ & $\begin{array}{c}\text { Vincristine- } \\
\text { carboplatin }\end{array}$ \\
\hline Subretinal seeding & $7(21.9 \%)$ & $8(24.2 \%)$ \\
At diagnosis & $3(9.4 \%)$ & $8(24.2 \%)$ \\
After chemoreduction & $9.3 \%$ & $9.1 \%$ \\
Vitreous seeding & & \\
\hline
\end{tabular}

Table 4 Treatments realized after the initial chemoreduction phase

\begin{tabular}{lcc}
\hline $\begin{array}{l}\text { Treatments after two cycles of } \\
\text { chemotherapy }\end{array}$ & $\begin{array}{l}\text { Etoposide- } \\
\text { carboplatin }\end{array}$ & $\begin{array}{c}\text { Vincristine- } \\
\text { carboplatin }\end{array}$ \\
\hline $\begin{array}{l}\text { Chemothermotherapy } \\
\text { (IV carboplatin alone and TTT) }\end{array}$ & 30 Eyes & 29 Eyes \\
TTT & 10 Eyes & 13 Eyes \\
Cryoapplication & 12 Eyes & 20 Eyes \\
Radioactive plaques & 2 Eyes & 3 Eyes \\
\hline
\end{tabular}

Abbreviations: IV, intravenous; TTT, transpupillary thermotherapy.

In the reference arm, 1 child did not receive any CTT, 1 child received one course, 3 children received 2 courses, 16 children received 3 courses, 6 children received 4 courses, none received 5 courses, and 1 received 6 courses for a relapsing lesion after 3 initial courses. In the experimental arm, 1 child did not receive CTT, none had just one course, 3 children had 2 courses, 12 children had 3 courses, 3 children had 4 courses, 1 child had 5 courses (for relapse after initial 3 courses), and finally 1 child received 6 courses ( 4 for relapsing lesions after 2 initial courses).

At the end of follow-up of 2 years for each child (and eye) included, the evaluation is realized and the number of failures is of 6 eyes in our reference arm and 10 in the 
Table 5 Systemic toxicities in each arm

\begin{tabular}{lcc}
\hline & $\begin{array}{c}\text { Etoposide- } \\
\text { carboplatin }\end{array}$ & $\begin{array}{c}\text { Vincristine- } \\
\text { carboplatin }\end{array}$ \\
\hline Red blood cell transfusion & 20 & 3 \\
Platelet transfusion & 23 & 5 \\
Grade IV neutropenia & 32 & 20 \\
Hospitalization between courses & 13 & 8 \\
\hline
\end{tabular}

new arm. In the reference arm, four eyes were enucleated, one eye received EBRT, and one was successively treated by EBRT and enucleation. In the vincristine-carboplatin arm, nine eyes required enucleation and one could be salvaged by EBRT.

In both arms, failures (EBRT and/or enucleation) are more common in group D eyes (3/6 failures in reference arm and 6/10 in experimental arm).

Systemic toxicity is described in Table 5. As expected, the hematological toxicity is higher in the reference arm but no severe or unexpected toxicity has been encountered and toxicity remains in the usual range of chemotherapy-induced toxicity at this age. No second cancer has been observed.

\section{Discussion}

This study describes the results of a prospective phase II randomized noncomparative study of chemoreduction by vincristine-carboplatin vs our standard treatment etoposide-carboplatin. This study was planned in order to decrease the short-, medium-, and long-term toxicities of etoposide.

Both arms are generally comparable in terms of initial characteristics at diagnosis. However, it must be underlined that the proportion of group D eyes was slightly higher in the 'new arm' (vincristine-carboplatin) that can have an impact on the success of conservative treatment. More subretinal seeding was also observed after neoadjuvant chemotherapy in this arm. This presentation is usually associated with a reduced eye preservation rate. The total carboplatin dose per course was based on previous publications and was very similar (respectively 600 and $560 \mathrm{mg} / \mathrm{m}^{2}$ in standard and experimental arm), even though it was administered in 3 days in the standard arm and 1 day in the experimental arm. ${ }^{8}$

The conservative strategy adopted after chemoreduction is comparable in the two arms and to our usual conservative management of retinoblastomas with children receiving local treatments and chemothermotherapy (one-drug chemotherapy associated to laser hyperthermia median of three cycles in each arm) for a maximum of 6 cycles of CTT (8 cycles of chemotherapy) for 3 children, all for relapsing lesions ( 1 in the reference arm and 2 in the experimental arm).
The total dose of carboplatin is therefore comparable to the dose used in the three-drug regimens and VP 16 is inferior in our standard arm.

Failures (need for enucleation or EBRT) were observed for 6 eyes in the reference arm and 10 in the new arm, just corresponding to the predefined threshold value for rejecting the null hypothesis (insufficient activity).

When the study was designed, the hypothesis was that failures would be $\sim 40 \%$ of treated eyes, which were the standard results for the EBRT studies for comparable patient cohorts. ${ }^{23}$ The study initially evaluated in 10 eyes the maximum number of failures to conclude the efficacy of the chemotherapy association used.

In the study we observed a failure for 10 eyes in the 'new arm', but only 6 in the reference arm. The initial hypothesis was therefore possibly more pessimistic than the obtained results in each arm. Nevertheless, the reference arm etoposide-carboplatin achieves better results, and the new vincristine-carboplatin arm seems insufficiently active when compared with the reference arm, and therefore it cannot be recommended for the chemoreduction phase.

Conservative management of retinoblastoma uses different strategies depending on each group. Intravenous chemotherapy is widely used in most of the protocols. Number of drugs and cycles vary. The association of three drugs for six cycles and the use of local adjuvant therapy is widely used by different groups. ${ }^{11,24,25}$

Our previous published study showed good results of our adopted strategy of conservative treatment associating intravenous neoadjuvant chemotherapy with two drugs (etoposide and carboplatin for two cycles) followed by $2-4$ cycles of carboplatin alone chemotherapy and laser thermotherapy (chemothermotherapy). Tumor control was obtained for $84 \%$ of the eyes and ocular preservation in $80 \%$ of the eyes. ${ }^{8}$

Other studies suggested that less chemotherapy could be used. The series showed that carboplatin and vincristine or even carboplatin alone may achieve good tumor control. ${ }^{12,26-28}$

This new study shows that deescalation, using vincristine instead of etoposide, seems not enough active to achieve satisfactory tumor control and eye preservation without EBRT. Furthermore, the very low risks of etoposide-induced secondary leukemia (at the doses we used, only for the initial two courses) should not defer physicians from its use in this indication.

During the progress of the study, other therapeutic approaches appeared for the conservative treatments of retinoblastoma, and direct intraarterial chemotherapy with melphalan or other drugs began to be used more widely, ${ }^{29-31}$ as well as the use of periocular chemotherapy and even intravitreal injections of cytotoxic drugs. ${ }^{15}$ The last data on intraarterial chemotherapy seem to show 
satisfactory eye preservation for first-line as well as second-line treatments. ${ }^{30,31}$ Short-term ocular toxicity may be seen with ischemic short-term complications being described. ${ }^{32,33}$ However, more follow-up is needed to evaluate long-term ocular preservation and toxicity of the technique.

Intravitreal injection of chemotherapy may be an interesting tool in the management of selected cases with active vitreous seeding. ${ }^{15}$

All these techniques can be combined to obtain the best tumor control, especially in the more advanced D group cases, these patients needing more treatments. Since the end of the study bilateral group D patients are treated by an association of six courses of three drugs chemotherapy regimen and local adjuvant therapies. Intravitreal injections are discussed on a case-to-case basis in the event of intravitreal seeding. Even though the use of intraarterial and intravitreal chemotherapy is very interesting, intravenous chemotherapy associated with local ocular treatments (cryoapplication and laser thermotherapy) remains a valid and efficient conservative management of retinoblastoma for a majority of patients.

Best ocular preservation and lowering the possible toxicities are the main concerns of conservative treatments.

Most of the failures are observed for group D eyes. For these advanced cases, treatment improvements are still needed in order to obtain a better ocular preservation with less possible systemic complications. For the less advanced stage, deescalation of therapy must be discussed.

This study does not allow to recommend the use of vincristine instead of etoposide in combination with carboplatin for the neoadjuvant treatment of intraocular retinoblastoma before chemothermotherapy and local adjuvant therapies.

\section{Summary}

What was known before

- Intravenous chemotherapy is the standard conservative option for conservative treatment of retinoblastoma. Protocols vary, with two- to three-drug regimen for 4-6 cycles.

What this study adds

- Randomized study of a two-drug regimen.

\section{Conflict of interest}

The authors declare no conflict of interest.

\section{Acknowledgements}

We thank the parents and the children who participated to the study and the parents and patients association
RETINOSTOP (www.retinostop.org) for their help in the preparation of the information and consent forms. We thank the pediatric oncologists of the SFCE (French society of cancer in children) who actively participated in the treatment of these patients. This study received a grant from the PHRC (Programme Hospitalier de Recherche Clinique) supported by the French Ministry of Health and the French National Cancer Institute. The study was declared as clinical trial at INCA (Institut National Cancer: French Institute for Cancer), as clinical study RECF 0151, and at the AFSSAPS (Agence Française de sécurité sanitaire des produits de santé: French agency for the security of drugs) as no. 040188.

\section{References}

1 Aerts I, Pacquement H, Doz F, Mosseri V, Desjardins L, Sastre $\mathrm{X}$ et al. Outcome of second malignancies after retinoblastoma: a retrospective analysis of 25 patients treated at the Institut Curie. Eur J Cancer 2004; 40(10): 1522-1529.

2 Kleinerman RA, Tucker MA, Tarone RE, Abramson DH, Seddon JM, Stovall M et al. Risk of new cancers after radiotherapy in long-term survivors of retinoblastoma: an extended follow-up. J Clin Oncol 2005; 23(10): 2272-2279.

3 Beck MN, Balmer A, Dessing C, Pica A, Munier F. First-line chemotherapy with local treatment can prevent externalbeam irradiation and enucleation in low-stage intraocular retinoblastoma. J Clin Oncol 2000; 18(15): 2881-2887.

4 Chantada GL, Fandino AC, Raslawski EC, Manzitti J, de Dávila MT, Casak SJ et al. Experience with chemoreduction and focal therapy for intraocular retinoblastoma in a developing country. Pediatr Blood Cancer 2005; 44(5): 455-460.

5 Cohen VM, Kingston J, Hungerford JL. The success of primary chemotherapy for group $\mathrm{D}$ heritable retinoblastoma. Br J Ophthalmol 2009; 93(7): 887-890.

6 Gombos DS, Kelly A, Coen PG, Kingston JE, Hungerford JL. Retinoblastoma treated with primary chemotherapy alone: the significance of tumour size, location, and age. Br J Ophthalmol 2002; 86(1): 80-83.

7 Lumbroso L, Doz F, Urbieta M, Levy C, Bours D, Asselain B et al. Chemothermotherapy in the management of retinoblastoma. Ophthalmology 2002; 109(6): 1130-1136.

8 Lumbroso-Le Rouic L, Aerts I, Levy-Gabriel C, Dendale R, Sastre $\mathrm{X}$, Esteve $\mathrm{M}$ et al. Conservative treatments of intraocular retinoblastoma. Ophthalmology 2008; 115(8): 1405-1410, 1410.e1401-1402.

9 Murphree AL, Villablanca JG, Deegan WF, Sato JK, Malogolowkin M, Fisher A et al. Chemotherapy plus local treatment in the management of intraocular retinoblastoma. Arch Ophthalmol 1996; 114: 1348-1356.

10 Schueler AO, Jurklies C, Heimann H, Wieland R, Havers W, Bornfeld N. Thermochemotherapy in hereditary retinoblastoma. Br J Ophthalmol 2003; 87(1): 90-95.

11 Shields CL, DePotter P, Himelstein BP, Shields JA, Meadows AT, Maris JM. Chemoreduction in the initial management of intraocular retinoblastoma. Arch Ophthalmol 1996; 114: 1330-1338.

12 Wilson MW, Rodriguez-Galindo C, Haik BG, Moshfeghi DM, Merchant TE, Pratt CB. Multiagent chemotherapy as 
neoadjuvant treatment for multifocal intraocular retinoblastoma. Ophthalmology 2001; 108(11): 2106-2114; discussion 2114-2105.

13 Abramson DH, Dunkel IJ, Brodie SE, Marr B, Gobin YP. Superselective ophthalmic artery chemotherapy as primary treatment for retinoblastoma (chemosurgery). Ophthalmology 2010; 117(8): 1623-1629.

14 Mallipatna AC, Dimaras H, Chan HS, Heon E, Gallie BL. Periocular topotecan for intraocular retinoblastoma. Arch Ophthalmol 2011; 129(6): 738-745.

15 Munier FL, Gaillard MC, Balmer A, Soliman S, Podilsky G, Moulin AP et al. Intravitreal chemotherapy for vitreous disease in retinoblastoma revisited: from prohibition to conditional indications. Br J Ophthalmol 2012; 96(8): 1078-1083.

16 Gombos DS, Hungerford J, Abramson DH, Kingston J, Chantada G, Dunkel IJ et al. Secondary acute myelogenous leukemia in patients with retinoblastoma: is chemotherapy a factor? Ophthalmology 2007; 114(7): 1378-1383.

17 Murphree ALIntraocular retinoblastoma: the case for a new group classification. In: AD Singh (ed). Ophthalmology Clinics of North America Vol. 18. Elsevier Saunders: Philadelphia, PA, USA, 2005, pp 41-53.

18 Jehanne M, Lumbroso-Le Rouic L, Savignoni A, Aerts I, Mercier G, Bours D et al. Analysis of ototoxicity in young children receiving carboplatin in the context of conservative management of unilateral or bilateral retinoblastoma. Pediatr Blood Cancer 2009; 52(5): 637-643.

19 Herson J, Carter SK. Calibrated phase II clinical trials in oncology. Stat Med 1986; 5(5): 441-447.

20 Rubinstein L, Crowley J, Ivy P, Leblanc M, Sargent D. Randomized phase II designs. Clin Cancer Res 2009; 15(6): 1883-1890.

21 Fleming TR. One-sample multiple testing procedure for phase II clinical trials. Biometrics 1982; 38(1): 143-151.

22 A'Hern RP. Sample size tables for exact single-stage phase II designs. Stat Med 2001; 20(6): 859-866.

23 Hungerford JL, Toma NM, Plowman PN, Kingston JE. External beam radiotherapy for retinoblastoma: I. Whole eye technique. Br J Ophthalmol 1995; 79(2): 109-111.

24 Gallie BL, Budning A, DeBoer G, Thiessen JJ, Koren G, Verjee $\mathrm{Z}$ et al. Chemotherapy with focal therapy can cure intraocular retinoblastoma without radiotherapy. Arch Ophthalmol 1996; 114: 1321-1328.
25 Shields CL, Shields JA, Needle M, de Potter P, Kheterpal S, Hamada A et al. Combined chemoreduction and adjuvant treatment for intraocular retinoblastoma. Ophthalmology 1997; 104(12): 2101-2111.

26 Rodriguez-Galindo C, Wilson MW, Haik BG, Merchant TE, Billups CA, Shah N et al. Treatment of intraocular retinoblastoma with vincristine and carboplatin. J Clin Oncol 2003; 21(10): 2019-2025.

27 Abramson DH, Lawrence SD, Beaverson KL, Lee TC, Rollins IS, Dunkel IJ. Systemic carboplatin for retinoblastoma: change in tumour size over time. Br J Ophthalmol 2005; 89(12): 1616-1619.

28 Gobin YP, Dunkel IJ, Marr BP, Francis JH, Brodie SE, Abramson DH. Combined, sequential intravenous and intra-arterial chemotherapy (bridge chemotherapy) for young infants with retinoblastoma. PLoS One 2012; 7(9): e44322.

29 Abramson DH, Dunkel IJ, Brodie SE, Kim JW, Gobin YP. A phase I/II study of direct intraarterial (ophthalmic artery) chemotherapy with melphalan for intraocular retinoblastoma initial results. Ophthalmology 2008; 115(8): 1398-1404, 1404.e1391.

30 Gobin YP, Dunkel IJ, Marr BP, Brodie SE, Abramson DH. Intra-arterial chemotherapy for the management of retinoblastoma: four-year experience. Arch Ophthalmol 2011; 129(6): 732-737.

31 Shields CL, Bianciotto CG, Jabbour P, Ramasubramanian A, Lally SE, Griffin GC et al. Intra-arterial chemotherapy for retinoblastoma: report no. 1, control of retinal tumors, subretinal seeds, and vitreous seeds. Arch Ophthalmol 2011; 129(11): 1399-1406.

32 Munier FL, Beck-Popovic M, Balmer A, Gaillard MC, Bovey E, Binaghi S. Occurrence of sectoral choroidal occlusive vasculopathy and retinal arteriolar embolization after superselective ophthalmic artery chemotherapy for advanced intraocular retinoblastoma. Retina 2011; 31(3): 566-573.

33 Shields CL, Bianciotto CG, Jabbour P, Griffin GC, Ramasubramanian A, Rosenwasser R et al. Intra-arterial chemotherapy for retinoblastoma: report no. 2, treatment complications. Arch Ophthalmol 2011; 129(11): 1407-1415.

Supplementary Information accompanies this paper on Eye website (http://www.nature.com/eye) 